\title{
HADRON STRUCTURE FUNCTIONS IN A CHIRAL QUARK MODEL
}

\author{
LEONARD GAMBERG*) \\ Department of Physics and Astronomy, University of Oklahoma, 440 West Brooks, \\ Norman, OK 73019 \\ HERBERT WEIGEL ${ }^{\ddagger}$ ) \\ Center for Theoretical Physics, Laboratory of Nuclear Science, Massachusetts Institute \\ of Technology, Cambridge, MA 02139
}

OKHEP-99-06, MIT-CTP-2913

\begin{abstract}
We present a consistent regularization procedure for calculating hadron structure functions in a bosonized chiral quark model. We find that the Pauli-Villars regularization scheme is most suitable. We also summarize the phenomenology of structure functions calculated in the valence quark approximation.
\end{abstract}

\section{Introduction}

The strong interaction contributions to the cross-sections in deep inelastic scattering are parameterized by the hadronic tensor $W_{\mu \nu}$. The associated form factors depend on the Lorentz-scalars $Q^{2}=-q^{2}$ and $x=Q^{2} / 2 p \cdot q$, with $p$ and $q$ being the momenta of the nucleon and the exchanged photon, respectively. The most convincing evidence for the quark-substructure of the nucleon stems from the socalled scaling behavior of these form factors implying that certain combinations thereof i.e. the structure functions, depend on $Q^{2}$ only logarithmically. Without bound-state wave functions in continuum QCD it has proven difficult to calculate the hadron structure functions from first principles. Fortunately the operator product expansion supplies an avenue to calculate nucleon structure functions: To leading twist (expansion in $1 / Q^{2}$ ) the moments of the structure functions factorize into non-perturbative and perturbative pieces. The former are tractable within effective low energy theories for QCD while the latter represent the logarithmic scaling violations which can be computed utilizing the renormalization group equations [1]. Alternatively one may first sum the series i.e. calculate the leading twist structure functions at the "model scale", $Q_{0}^{2}$, and subsequently incorporate the radiative correction using the DGLAP evolution procedure.

In a field theory calculation the most suitable starting point to compute hadron structure functions is the forward Compton amplitude for hadron photon scattering

$$
T_{\mu \nu}(p, q ; s)=i \int d^{4} \xi e^{i q \cdot \xi}\left\langle p, s\left|T\left\{J_{\mu}(\xi) J_{\nu}^{\dagger}(0)\right\}\right| p, s\right\rangle
$$

*) Talk presented by L. Gamberg at "Symmetry and Spin" PRAHA-SPIN99, Sept. 6-11, 1999.

‡) Heisenberg-Fellow 
since the time-ordered correlation function of electro-magnetic currents, $J_{\mu}$ can be calculated in the functional integral formalism. In turn the hadronic tensor is obtained from its absorptive contribution,

$$
W_{\mu \nu}(p, q ; s)=\frac{1}{2 \pi} \operatorname{Im} T_{\mu \nu}(p, q ; s) .
$$

The structure functions are then extracted from $W_{\mu \nu}$ by adopting the Bjorken limit, $Q^{2} \rightarrow \infty$ with $x$ fixed.

\section{Generalities on the Structure Functions in the NJL-Model}

The results and discussions presented in this section are based on ref. [2]. The interaction in the Nambu-Jona-Lasinio (NJL) model is a chirally invariant four fermion coupling, with coupling constant $G$. Employing functional integration techniques we may recast it into an effective meson theory [3]. What remains is to properly regularize the UV divergence of the resulting determinant of the Dirac operator in the background of the meson fields. We utilize the Pauli-Villars (PV) regularization scheme which provides a formulation of the bosonized NJL model completely in Minkowski space 画. This is suitable when applying Cutkosky's rules to extract the hadronic tensor via (2). In addition the PV scheme yields the proper scaling of the structure functions since the UV cut-off is additive in both the loop momenta and the (constituent) quark masses.

In the NJL chiral soliton model regularizing the nucleon structure functions has only recently been addressed in a satisfactory manner [2]. In the first calculations [5, 6, 7, contributions from the polarized vacuum to the structure functions were approximated within the (constituent) valence quark approximation. This has been motivated by the observation that the regularized vacuum contributions to static nucleon properties are small once the self-consistent soliton solution is determined. Later approaches including vacuum contributions imposed a single subtraction regularization scheme at the level of (constituent) quark distributions [8, 9]. The regularization attributed to a given structure function was conjectured to equal that of the corresponding sum rule associated with static nucleon properties. This approach is obviously inadequate for structure functions whose sum rules are not associated with a matrix element of a local operator. Also, the single subtraction scheme leaves the gap-equation determining the VEV, $m=\langle S\rangle$ undefined.

To calculate the electro-magnetic current operator appearing in the Compton tensor (11) we introduce sources $v_{\mu}$ which are conjugate to this current

$$
\begin{aligned}
i \mathbf{D} & =i \not \partial-\left(S+i \gamma_{5} P\right)+\mathcal{Q} \psi=: i \mathbf{D}^{(\pi)}+\mathcal{Q} \psi \\
i \mathbf{D}_{5} & =-i \not \partial-\left(S-i \gamma_{5} P\right)-\mathcal{Q} \psi=: i \mathbf{D}_{5}^{(\pi)}-\mathcal{Q} \psi,
\end{aligned}
$$

with $\mathcal{Q}=\operatorname{diag}(2 / 3,1 / 3)$ denoting the quark charge matrix. $S$ and $P$ are respectively scalar and pseudo-scalar matrix valued fields. In addition to the common Dirac operator $\mathbf{D}$, is the $\gamma_{5}$-odd Dirac operator $\mathbf{D}_{5}$, which in Euclidean space would be 
associated to the Hermitian conjugate of $\mathbf{D}$. The bosonized and PV regularized form of the NJL-model then reads $\left(\sum_{i} c_{i}=\sum_{i} c_{i} \Lambda_{i}^{2}=0, \Lambda_{0}=0, c_{0}=1\right)$,

$$
\begin{aligned}
& \mathcal{A}_{\mathrm{NJL}}\left[v_{\mu}\right]=\frac{1}{4 G} \int d^{4} x \operatorname{tr}\left[S^{2}+P^{2}+2 m_{0} S\right] \\
&-i \frac{N_{C}}{2} \sum_{i=0}^{2} c_{i} \operatorname{Tr} \log \left[-\mathbf{D D}_{5}+\Lambda_{i}^{2}-i \epsilon\right]-i \frac{N_{C}}{2} \operatorname{Tr} \log \left[-\mathbf{D}\left(\mathbf{D}_{5}\right)^{-1}-i \epsilon\right] .
\end{aligned}
$$

The fact that the last part of (5) is not regularized ensures that the ABJ-anomaly is completely recovered. The time ordered matrix element of the currents is obtained by expanding the action to quadratic order in the sources $v_{\mu}$. In ref. 2 we have shown that considerable simplifications arise in the Bjorken limit; in particular the propagator between two insertions of the sources becomes that of a free quark, yielding the action

$$
\begin{aligned}
\mathcal{A}^{(2, v)}= & \frac{i N_{C}}{4} \sum_{i=0}^{2} c_{i} \operatorname{Tr}\left\{\left(\mathbf{D}^{(\pi)} \mathbf{D}_{5}^{(\pi)}-\Lambda_{i}^{2}\right)^{-1}\left[\mathcal{Q}^{2} \psi(\not \partial)^{-1} \psi \mathbf{D}_{5}^{(\pi)}-\mathbf{D}^{(\pi)}\left(\psi(\not \partial)^{-1} \psi\right)_{5} \mathcal{Q}^{2}\right]\right\} \\
& +\frac{i N_{C}}{4} \operatorname{Tr}\left\{\left(-\mathbf{D}^{(\pi)} \mathbf{D}_{5}^{(\pi)}\right)^{-1}\left[\mathcal{Q}^{2} \psi(\not \partial)^{-1} \psi \mathbf{D}_{5}^{(\pi)}+\mathbf{D}^{(\pi)}\left(\not(\not \partial)^{-1} \psi\right)_{5} \mathcal{Q}^{2}\right]\right\} .
\end{aligned}
$$

The above mentioned decomposition of the action into regularized and unregularized pieces has enforced the specification $\left(S_{\mu \rho \nu \sigma}=g_{\mu \rho} g_{\nu \sigma}+g_{\rho \nu} g_{\mu \sigma}-g_{\mu \nu} g_{\rho \sigma}\right)$,

$\gamma_{\mu} \gamma_{\rho} \gamma_{\nu}=S_{\mu \rho \nu \sigma} \gamma^{\sigma}-i \epsilon_{\mu \rho \nu \sigma} \gamma^{\sigma} \gamma^{5} \quad$ while $\quad\left(\gamma_{\mu} \gamma_{\rho} \gamma_{\nu}\right)_{5}=S_{\mu \rho \nu \sigma} \gamma^{\sigma}+i \epsilon_{\mu \rho \nu \sigma} \gamma^{\sigma} \gamma^{5}$.

The latter reduction in $(\nabla)$ which is reflected in that of $\left(\psi(\not \partial)^{-1} \psi\right)_{5}$, arises in order that the regularization of the structure functions be consistent with the corresponding sum rules ${ }^{1}$ ). Finally from (6) we calculate the bilocal current correlation function and apply Cutkosky's rules to obtain the absorptive part (2).

The pion fields are identified as the angular variables on the chiral circle, $S+i P=m \exp (i g \vec{\tau} \cdot \vec{\pi} / m)$ with the constituent quark mass being the vacuum expectation value (VEV) of the scalar field, $m=\langle S\rangle$. The quark-pion coupling constant is extracted from the normalization of the pion field yielding

$$
\frac{1}{g^{2}}=\left.4 N_{C} \frac{d}{d q^{2}} \int_{0}^{1} d x\left[q^{2} \Pi\left(q^{2}, x\right)\right]\right|_{q^{2}=m_{\pi}^{2}}
$$

where

$$
\Pi\left(q^{2}, x\right)=-i \sum_{i=0}^{2} c_{i} \frac{d^{4} k}{(2 \pi)^{4}}\left[-k^{2}-x(1-x) q^{2}+m^{2}+\Lambda_{i}^{2}-i \epsilon\right]^{-2}
$$

is the pion-polarization function parameterizing the quark loop. Upon expanding $\mathbf{D}^{(\pi)}$ and $\mathbf{D}_{5}^{(\pi)}$ up to quadratic order in the pion fields we obtain the virtual Compton amplitude for pion-photon scattering and extract the pion structure function

$\left.{ }^{1}\right)$ This issue is not unique to the PV regularization scheme; in fact all schemes which regularize the sum $\log (\mathbf{D})+\log \left(\mathbf{D}_{5}\right)$ but not the difference $\log (\mathbf{D})-\log \left(\mathbf{D}_{5}\right)$ require this treatment. 
to be

$$
F(x)=\left.\frac{5}{9}\left(4 N_{C} g^{2}\right) \frac{d}{d q^{2}}\left[q^{2} \Pi\left(q^{2}, x\right)\right]\right|_{q^{2}=m_{\pi}^{2}} .
$$

In the chiral limit, $m_{\pi}=0$, the pion structure function turns out to be independent of $x$ and simply reproduces the pion-quark coupling (8). Although this result has been known [10] it serves to verify the approach defined by the expansion (6).

In this model the nucleon emerges as a chiral soliton [11. The starting point for constructing the static soliton is the Dirac Hamiltonian, $h$ is defined from (3),

$$
i \mathbf{D}^{(\pi)}=\beta\left(i \partial_{t}-h\right) \quad \text { and } \quad i \mathbf{D}_{5}^{(\pi)}=\left(-i \partial_{t}-h\right) \beta .
$$

It is diagonalized for the well-known hedgehog configuration of the background meson fields,

$$
h \Psi_{\alpha}=\epsilon_{\alpha} \Psi_{\alpha} \quad \text { with } \quad h=\vec{\alpha} \cdot \vec{p}+m \beta \exp \left(i \overrightarrow{\hat{r}} \cdot \vec{\tau} \gamma_{5} \Theta(r)\right),
$$

yielding eigen-spinors $\Psi_{\alpha}$ and energy eigenvalues $\epsilon_{\alpha}$. For this mesonic configuration the action functional is expressed as a regularized sum over the eigenvalues $\epsilon_{\alpha}$. This yields an energy functional, $E[\Theta]$ which equals, to leading order in $1 / N_{C}$, the nucleon mass. The minimization of $E[\Theta]$ determines the localized profile function $\Theta(r)$, self-consistently. Nucleon states are generated by introducing collective coordinates for the position of the soliton as well as its orientation in coordinate and flavor spaces. Canonical quantization of these coordinates yields the nucleon states which enter the matrix elements (11). Finally the structure functions are obtained by contracting the corresponding hadronic tensor (2) with pertinent projectors. As an example we list the resulting expression for the polarized structure function $g_{1}$,

$$
\begin{aligned}
g_{1}(x)=- & i \frac{M_{N}[\Theta] N_{C}}{36} \int \frac{d \omega}{2 \pi} \sum_{\alpha} \int d^{3} \xi \int \frac{d \lambda}{2 \pi} \mathrm{e}^{i M_{N}[\Theta] x \lambda} \\
\times & \left\{\left(\sum_{i=0}^{2} c_{i} \frac{\omega+\epsilon_{\alpha}}{\omega^{2}-\epsilon_{\alpha}^{2}-\Lambda_{i}^{2}+i \epsilon}\right)_{\mathrm{p}}\left\langle N\left|I_{3}\right| N\right\rangle\right. \\
\times & \left.\times \Psi_{\alpha}^{\dagger}(\vec{\xi}) \tau_{3}\left(1-\alpha_{3}\right) \gamma_{5} \Psi_{\alpha}\left(\vec{\xi}_{\lambda}^{+}\right) \mathrm{e}^{-i \omega \lambda}+\Psi_{\alpha}^{\dagger}(\vec{\xi}) \tau_{3}\left(1-\alpha_{3}\right) \gamma_{5} \Psi_{\alpha}\left(\vec{\xi}_{\lambda}^{-}\right) \mathrm{e}^{i \omega \lambda}\right] \\
& \quad-\frac{30}{\alpha^{2}[\Theta]}\left[\frac{i \lambda}{4}\left(\frac{\omega+\epsilon_{\alpha}}{\omega^{2}-\epsilon_{\alpha}^{2}+i \epsilon}\right)_{\mathrm{p}}\right. \\
\times & {\left[\Psi_{\alpha}^{\dagger}(\vec{\xi}) \tau_{3}\left(1-\alpha_{3}\right) \gamma_{5} \Psi_{\alpha}\left(\vec{\xi}_{\lambda}^{+}\right) \mathrm{e}^{-i \omega \lambda}-\Psi_{\alpha}^{\dagger}(\vec{\xi}) \tau_{3}\left(1-\alpha_{3}\right) \gamma_{5} \Psi_{\alpha}\left(\vec{\xi}_{\lambda}^{-}\right) \mathrm{e}^{i \omega \lambda}\right] } \\
& +\sum_{\beta}\left(\frac{\left(\omega+\epsilon_{\alpha}\right)\left(\omega+\epsilon_{\beta}\right)}{\left(\omega^{2}-\epsilon_{\alpha}^{2}+i \epsilon\right)\left(\omega^{2}-\epsilon_{\beta}^{2}+i \epsilon\right)}\right)_{\mathrm{p}}\left\langle\alpha\left|\tau_{3}\right| \beta\right\rangle \\
\times & {\left.\left[\Psi_{\beta}^{\dagger}(\vec{\xi})\left(1-\alpha_{3}\right) \gamma_{5} \Psi_{\alpha}\left(\vec{\xi}_{\lambda}^{+}\right) \mathrm{e}^{-i \omega \lambda}+\Psi_{\beta}^{\dagger}(\vec{\xi})\left(1-\alpha_{3}\right) \gamma_{5} \Psi_{\alpha}\left(\vec{\xi}_{\lambda}^{-}\right) \mathrm{e}^{i \omega \lambda}\right]\right\} }
\end{aligned}
$$


where $\vec{\xi}_{\lambda}^{ \pm}=\vec{\xi} \pm \lambda \hat{e}_{3}$ label shifted coordinates. The subscript 'p' indicates that the spectral integral is restricted to those values of $\omega$ which cause the respective denominators of the spectral functions to vanish. Similar expressions are obtained for $f_{1}$ and $g_{2}$ [2]. For the polarized structure functions is important to remark that the isoscalar contribution, which is next to leading order in $1 / N_{C}$ does not undergo regularization while the leading order isovector contribution does. As in the study of static nucleon properties the NJL model we find that either the isoscalar or isovector contributions to any structure function become regularized, but not both. The result that the isovector part is regularized was anticipated because we know how to relate the zeroth moments to nucleon charges 2 . With regard to the unpolarized structure function $f_{1}(x)$ we find the reverse situation: a regularized leading order isoscalar and an unregularized next to leading order isovector contribution. The latter piece enters the Gottfried sum rule implying that it is unregularized ${ }^{2}$ ).

The verification of the sum rules, which relate moments of the structure functions to static properties of hadrons, is lengthy but straightforward. Essentially it can be traced back to the expansion (6) which can be formally interpreted as a first order expansion in the operator $\mathcal{Q}^{2}\left(\not(\not \partial)^{-1} \psi\right)_{5}$. Such first order expansions are related to static properties.

\section{Numerical Results for the Nucleon Structure Functions}

Numerical results are still unavailable for the structure functions e.g. (13) as projected from the consistently regularized hadronic tensor. Thus, we confine ourselves to presenting results from the valence quark approximation [5, 6]. To compare the model structure functions with data, we calculate at the intrinsic scale of the model in the nucleon's rest frame $(\mathrm{RF})$, transform them to the infinite momentum frame (IMF) and evolve the results to scales commensurate with the data. We note that the Bjorken limit singles out the IMF which is preferred for structure functions calculated from extended field configurations such as the chiral soliton because in this frame this class of structure functions possesses proper support 12. The DGLAP evolution determines this scale parameter $Q_{0}^{2}$ at which the model approximates QCD.

The unpolarized structure functions are calculated from the symmetric portion of the hadronic tensor, $W_{\mu \nu}+W_{\nu \mu}$. In Fig. 1 we display the result of the structure functions entering the Gottfried sum rule for $e-N$ scattering as calculated in the RF and in the IMF. The DGLAP evolution determines our scale to be, $Q_{0}^{2} \approx 0.4 \mathrm{GeV}^{2}$. The resulting structure function reproduces the gross features of the experimental data. In addition depending on the other model parameter $m$, the Gottfried sum rule, $S_{G}=\int d x\left(F_{2}^{\mathrm{ep}}-F_{2}^{\mathrm{en}}\right) / x$ ranges between $0.26-0.29$ which exhibits the desired deviation from the naive quark model prediction of $1 / 3$ and is in reasonable agreement with the experimental range, $0.235 \pm 0.026$ [13].

\footnotetext{
${ }^{2}$ ) This is in contrast to previous studies [8, 9] where this sum rule was regularized based upon the assumption that it behaved equivalently to the Adler sum under regularization.
} 
L. Gamberg and H. Weigel
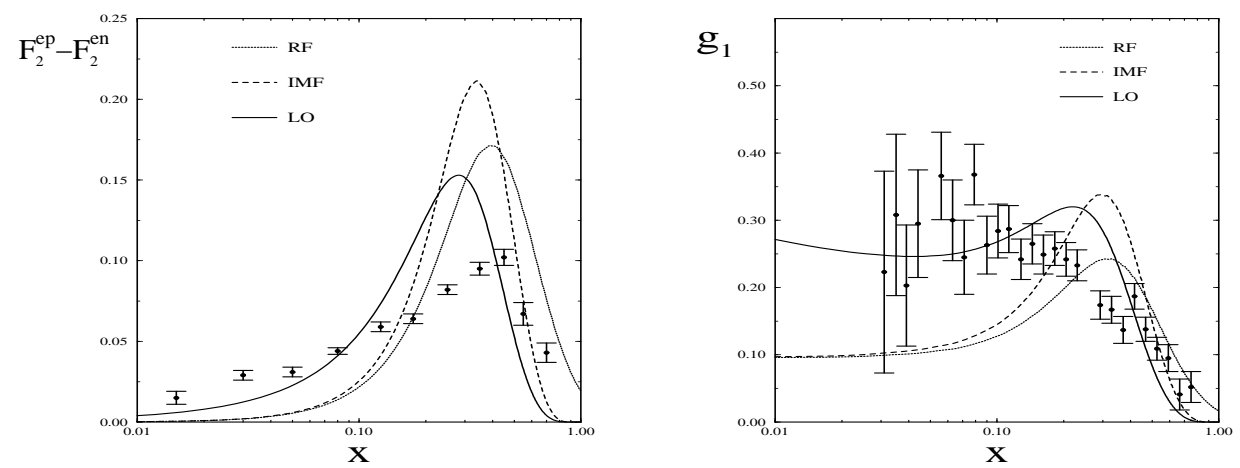

Fig. 1. Left Panel: The unpolarized structure function entering the Gottfried sum rule: $\mathrm{RF}$, IMF and leading order QCD evolution to $Q^{2}=3 \mathrm{GeV}^{2}$, with full $1 / N_{c}$ contribution; see []. Right Panel: $g_{1}(x)$.
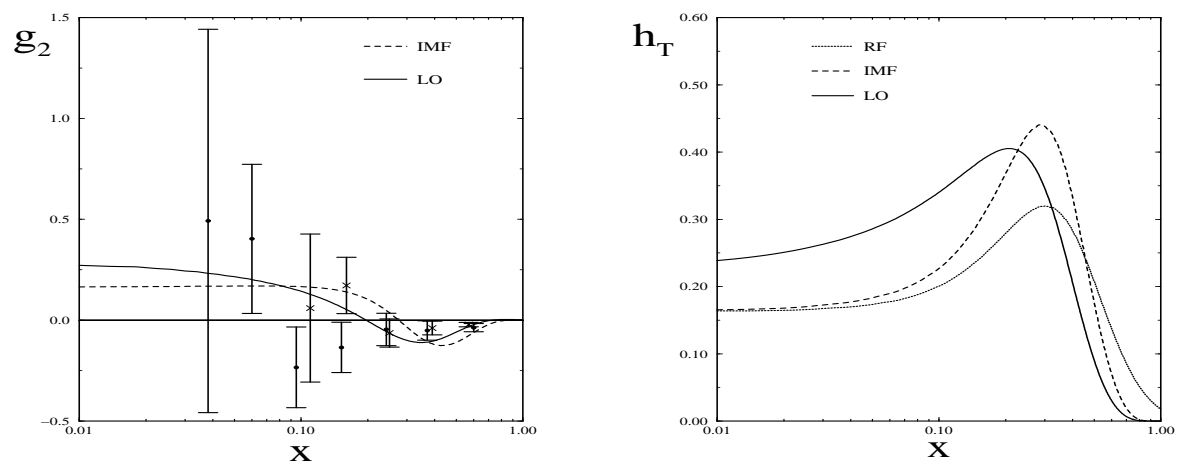

Fig. 2. Left: $g_{2}^{p}\left(x, Q^{2}\right)=g_{2}^{W W(p)}\left(x, Q^{2}\right)+\bar{g}_{2}^{p}\left(x, Q^{2}\right)$ evolved from $Q_{0}^{2}=0.4 \mathrm{GeV}^{2}$ to $Q^{2}=5.0 \mathrm{GeV}^{2}$. Right Panel: Chiral odd structure function $h_{T}^{p}\left(x, Q^{2}\right)$.

The spin-polarized structure functions $g_{1}$ and $g_{2}$ are extracted from the antisymmetric combination, $W_{\mu \nu}-W_{\nu \mu}$. Results are shown in Fig. 2 and are compared with the data from SLAC 14. In particular we note the sizeable twist two and three contributions. The twist two portion is given by the Wandzura-Wilczek contribution, the first two terms in

$$
g_{2}\left(x, Q^{2}\right)=-g_{1}\left(x, Q^{2}\right)+\int_{x}^{1} \frac{d y}{y} g_{1}\left(x, Q^{2}\right)+\bar{g}_{2}\left(x, Q^{2}\right),
$$

where $\bar{g}_{2}$ denotes the twist three contribution. In the evolution of the twist-2 portion we have restricted ourselves to the leading order in $\alpha_{s}$ since the next to leading order evolution of the twist- $3 \bar{g}_{2}\left(x, Q^{2}\right)$ is not known (The twist-3 piece is known only in the large $1 / N_{\mathrm{c}}$ limit 15].). In ref. [2 the Bjorken $\int d x\left(g_{1}^{p}(x)-g_{1}^{n}(x)\right)=g_{A} / 6$ and Burkhardt-Cottingham $\int d x g_{2}(x)=0$ sum rules have been explicitly verified.

Having calculated the spin (un)polarized structure functions, $f_{1}$ and $g_{1}$ and 
their constituent quark components we investigate the chiral odd flavor distributions and their charge weighted average nucleon "structure functions" 16. Here we confine ourselves to the presentation of results relevant in the context of the Soffer inequality [17. This inequality relates the nucleon chiral odd quark distribution functions to both the unpolarized $f_{1}^{(u)}\left(x, Q_{0}^{2}\right)$ and polarized $g_{1}^{(u)}\left(x, Q_{0}^{2}\right)$ chiral even counter-parts.

$$
f_{1}^{(u)}\left(x, Q^{2}\right)+g_{1}^{(u)}\left(x, Q^{2}\right) \geq 2 h_{T}^{(u)}\left(x, Q^{2}\right) .
$$

The superscript refers to the flavor combination which projects onto up-quark quantum numbers. Note again, that this projection refers to the constituent quarks which contain some non-perturbative gluonic distributions. Fig. 3 clearly demonstrates that the inequality is satisfied at the model scale.
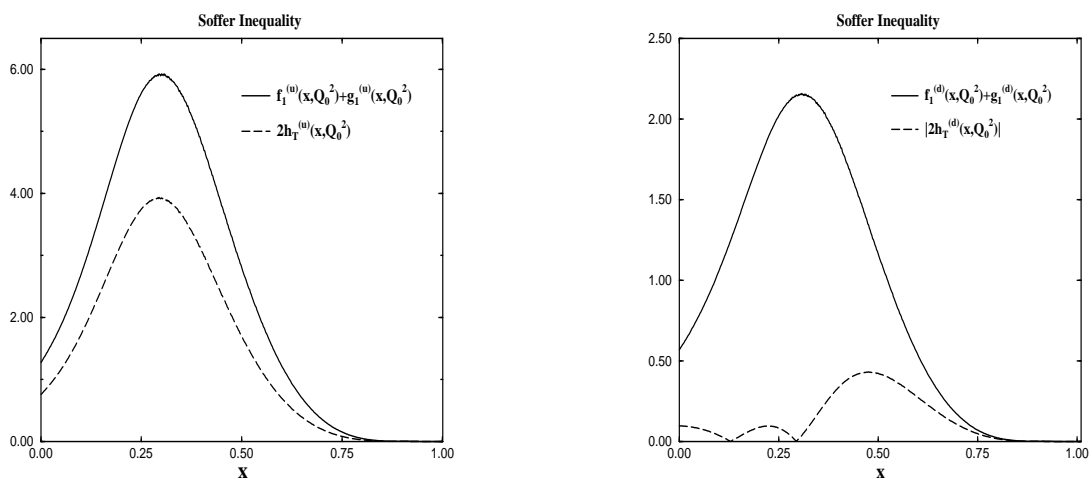

Fig. 3. Left Panel: The Soffer inequality for the chiral even combination in the IMF (solid line) for the up-quark distributions and the chiral odd structure function $2 h_{T}^{(u)}\left(x, Q_{0}^{2}\right)$ (long-dashed line). Right Panel: Down quark distributions.

\section{Conclusions}

We have summarized our results on the consistent regularization scheme in calculating hadron structure functions in the bosonized NJL chiral soliton model where baryons emerge as chiral solitons. We have calculated the Compton amplitude in the Bjorken limit from the PV regularized action functional with the constraint of preserving the anomaly structure of QCD. Introducing pertinent external sources enables the unambiguous identification of the quark currents which in turn allowed us to calculate the bilocal current correlation function entering the forward Compton amplitude. This scheme satisfies the leading order scaling properties of nucleon structure functions. The sum rules for both polarized and unpolarized are fulfilled because in the associated moments Cutkosky's rules extract - similar to the Cauchy 
integrals - the matrix elements associated with static nucleon properties [2]. These results represent the first step towards a fully consistent calculation of structure functions in the NJL model. We have also summarized our results in the valence quark approximation. Due the sizeable contribution from this state to all nucleon properties in the NJL model, not surprisingly, this approximation reproduces the general trends of the data on structure functions.

\section{Acknowledgement}

We gratefully acknowledge contributions from our co-workers H. Reinhardt and E. Ruiz Arriola. L.G. wishes to thank the organizers of SPIN-99 workshop for their efforts and hospitality and to K.A. Milton for support on this project. This work is supported by funds provided by the U.S. Department of Energy (D.O.E.) \#DE-FG03-98ER41066, and \#DF-FC02-94ER40818, and the Deutsche Forschungsgemeinschaft (DFG) under contract We $1254 / 3-1$.

\section{References}

[1] R. L. Jaffe and G. G. Ross, Phys. Lett. 93 B (1980) 313;

R. L. Jaffe and X. Ji, Phys. Rev. D43 (1991) 724.

[2] H. Weigel, E. Ruiz Arriola and L. Gamberg, Nucl. Phys. B560 (1999) 383.

[3] D. Ebert and H. Reinhardt, Nucl. Phys. B271 (1986) 188.

[4] E. Ruiz Arriola and L. L. Salcedo, Nucl. Phys. A590 (1995) 703.

[5] H. Weigel, L. Gamberg and H. Reinhardt, Mod. Phys. Lett. A11 (1996) 3021; Phys. Lett. B399 (1997) 287.

[6] H. Weigel, L. Gamberg and H. Reinhardt, Phys. Rev. D55 (1997) 6910.

[7] O. Schröder, H. Reinhardt and H. Weigel, Phys. Lett. B439 (1998) 398; Nucl. Phys. A651 (1999) 174.

[8] D. I. Diakonov et al., Nucl. Phys. B480 (1996) 341, Phys. Rev. D56 (1997) 4069.

[9] M. Wakamatsu and T. Kubota, Phys. Rev. D57 (1998) 5755; P. V. Pobylitsa et al., Phys. Rev. D59 (1999) 034024.

[10] R. M. Davidson and E. Ruiz Arriola, Phys. Lett. B348 (1995) 163; T. Frederico and G. A. Miller, Phys. Rev. D50 (1994) 210.

[11] R. Alkofer, H. Reinhardt and H. Weigel, Phys. Rep. 265 (1996) 139;

C. V. Christov et al., Prog. Part. Nucl. Phys. 37 (1996) 91.

[12] R. L. Jaffe, Ann. Phys. (NY) 132 (1981) 32;

L. Gamberg, H. Reinhardt and H. Weigel, Int. J. Mod. Phys. A13 (1998) 5519.

[13] M. Arneodo, et al., (NMC), Phys. Rev. D50 (1994) R1.

[14] K. Abe et al., Phys. Rev. Lett. 76 (1996) 587.

[15] A. Ali, V. M. Braun, and G. Hiller, Phys. Lett. B266 (1991) 117;

I. I. Balitskii, V. M. Braun, Y. Koike, and K. Tanaka, Phys. Rev. Lett. 77 (1996) 3078.

[16] L. Gamberg, H. Reinhardt and H. Weigel, Phys. Rev. D58 (1998) 054014.

[17] J. Soffer, Phys. Rev. Lett. 74 (1995) 1292; G. R. Goldstein, R. L. Jaffe, and X. Ji, Phys. Rev. D52 (1995) 5006. 